\title{
Evaluating Plug-In Vehicles (Plug-in Hybrid and Battery Electric Vehicles) Using Standard Dynamometer Protocols
}

\author{
Michael Duoba, Henning Lohse-Busch, Eric Rask \\ Argonne National Laboratory, 9700 South Cass Ave., Lemont, IL 60439, mduoba@anl.gov
}

\begin{abstract}
New plug-in vehicles offer advantages in fuel saving and lower exhaust emissions by adding new advanced-technology powertrain components. With this new complexity come new challenges in characterizing these vehicles using standardized testing methods. Over the last several years, many testing experts have developed new methods for testing battery electric vehicles (BEVs) and plug-in hybridelectric vehicles (PHEVs). New instrumentation hardware and techniques are outlined in this paper. Metrics and calculation methods were developed to suit the plug-in aspect and operational nature of the vehicles. New BEV test methods were developed and validated using a 2012 Nissan Leaf BEV. Analysis of the results showed that efficiency and range determined from the old methods could be reliably reproduced with the new procedures and methods. Likewise new PHEV test procedures were validated using a 2012 Chevy Volt PHEV and an aftermarket PHEV-converted Prius. The results of the test program show that the end-of-test criterion presented as the "alternate" method in SAE J1711 is the robust choice to determine the transition from depleting to sustaining. The Utility Factor method of calculating final results worked well for both PHEVs. Sample post-processing calculations are shown for the urban-cycle testing. Various PHEV range definitions and fuel and electricity usage rates are given with and without utility factor weighting.
\end{abstract}

Keywords: efficiency, energy consumption, EV (electric vehicle), PHEV (plug in hybrid electric vehicle)

\section{Introduction}

A plug-in electric vehicle (PEV) is any vehicle that uses off-board electric energy for propulsion, such as a battery electric vehicle (BEV) or plugin hybrid electric vehicle (PHEV). Argonne has considerable experience testing PEVs in its inhouse dynamometer test facilities and at other partner facilities. The authors led the 2010 revision of SAE J1711[1]-recommended practice for testing hybrid electric vehicles (HEVs) and PHEVs, and are key contributors to the revision of SAE J1634 [2] test procedures for BEVs. Past testing at Argonne has included aftermarket and prototype PHEVs and BEVs. After doing much procedure development on prototype PHEVs and BEVs, Argonne has had the recent opportunity to test production vehicles according to the new PEV standards. The three vehicles examined in this paper are 1) the Nissan Leaf BEV, 2) the Chevy Volt PHEV, and for comparison, 3) a secondgeneration Prius converted to PHEV operation. Having vehicles with production-level robustness and test-to-test repeatability provides an opportunity to demonstrate and validate test methodology. Actual vehicle data make it easier to 
illustrate how the data are processed in order to present final results. Fuel and electric consumption are examined for each cycle tested.

\section{Background}

Although the focus of this paper is on the urban test described below (and the highway [HWY] test, for the Leaf), normal benchmark testing includes the HWY test, the Supplemental Federal Test Procedure cycle (US06), and other ambienttemperature cycle tests. Each cycle is associated with a particular test procedure that includes unique initial conditions and procedures.

\subsection{Urban Driving Dynamometer Schedule Testing}

The Urban Driving Dynamometer Schedule (UDDS) test is also called the Federal Test Procedure (FTP). It is the basis for urban fuel economy and emissions certification. The test includes running a 1372-s time/speed trace (the UDDS) on a dynamometer (in road-load mode) twice. The first UDDS cycle is referred to as the "cold-start UDDS" because the vehicle starts and runs the cycle on the dynamometer after it has been sitting at room temperature overnight (1236 h), i.e., "soaking." The second UDDS cycle, performed after soaking for $10 \mathrm{~min}$, is referred to as the "hot-start UDDS." Conventional vehicles are studied with an abbreviated version of this test that does away with the end of the hot-start UDDS because it is assumed to be redundant with the end of the cold-start UDDS. This shortcut approach is not considered when testing HEVs; instead, both cycles are tested to completion. The fuel consumption and emissions results from the two UDDS cycles are combined using the following weighting:

$$
Y_{\text {weighted }}=0.43 \times Y_{\text {cold }}+0.57 \times Y_{\text {hot }} \text {, }
$$

where $Y$ is the emissions or fuel consumption per distance.

Since emission rates are very sensitive to initial start conditions, the weighting represents the contributions of cold-start operation after the vehicle sits for a long period of time compared to more frequent hot-starts throughout any given day.

\subsection{Highway Testing}

Whereas the UDDS testing includes specific initial conditions to capture transient warm-up operation, the HWY testing procedures specify warmstabilized operation-representing a long enough trip on the highway to justify neglecting the initial warm-up conditions. Two HWY cycles are run with no pause in between. The fuel consumption is only recorded during the second, stabilized HWY cycle. The first cycle is considered the preparation or "prep" cycle and is not part of the measured fuel consumption.

\subsection{US06 Testing}

Like HWY cycle testing, the US06 is a hotstabilized test procedure. The prep cycle is typically another US06 cycle (but other prep cycles can be run according to the Code of Federal Regulations [3]). Like the HWY test, only the results of the cycle following the prep cycle are used for the final results.

\subsection{SC03 and Cold UDDS Testing}

Two additional tests are common in certification vehicle testing: the SC03 test and the Cold UDDS test. The SC03 test is run with the test cell at elevated temperatures $\left(35^{\circ} \mathrm{C}\right)$ and with solar lamps emulating the heat load from the sun $\left(850 \mathrm{~W} / \mathrm{m}^{2}\right)$. The Cold UDDS test is run at low temperature $\left(-7^{\circ} \mathrm{C}\right)$ with basically the same procedures as the conventional UDDS test procedure. These tests were recently included as part of the U.S. Environmental Protection Agency's fuel economy estimations [4]. However, approaches taken to estimate real-world fuel economy on the basis of standard test results for advanced vehicles like BEVs and HEVs have been met with significant challenges [5]. More discussion of this aspect will be presented later in the paper.

At the time of publication, the upgrade of Argonne's dynamometer test facility to test hot and cold procedures was not fully commissioned, and results under these conditions were not yet available.

\subsection{Additional Test Requirements for PEVs}

Standard test protocols required additions that are specific to PEVs. The challenge was to integrate these additional requirements without changing the spirit of the test objectives or the context of the results. 


\subsubsection{Instrumentation for PEV Testing}

With the introduction of an additional source of propulsion energy (electricity), BEVs and all HEVs (including PHEVs) must be tested with equipment that measures the electric energy contribution to driving. The measurements must be made during testing (discharge) and, for PEVs, during recharge. Several instrumentation companies offer acceptable power analyzers for this task. Many test labs have used the Hioki 3193 [6], which has six channels of current and voltage inputs. The 4-channel Hioki 3390 (or $3390-10)$ [7,8] is the latest instrument that is marketed explicitly for applications such as electrified-vehicle testing.

The important minimum parameters are voltage, current, integrated Ah and integrated Wh. It is possible to run tests without directly measuring voltage if accurate information is available on the vehicle network bus. The integrated Ah must be measured at a high frequency. This is a critical function of the power analyzer.

The battery power and energy measurement accuracy necessary for chassis dynamometer testing has not been studied in enough detail to develop a specific requirement to date. It is hoped that more attention will be given to this issue soon. Argonne has done some investigations of accuracy and drift and found that errors can exist. Careful attention to the orientation of the current probe and to zeroing the meter frequently will help reduce these errors.

\subsubsection{Recharging PEVs}

Recharging PEVs is a new test requirement in chassis dynamometer testing. The UDDS test requires an overnight soak before the test. Test engineers have agreed that the soak period would be the most appropriate time to perform the recharge. Doing recharges serially may not always be representative of normal operation and may limit the flexibility to keep within the 36-h soak window requirement. The minimum soak time is $12 \mathrm{~h}$ and the procedures for recharge parallel this interval by recommending a minimum recharge time of $12 \mathrm{~h}$. It is likely that almost every vehicle will be able to recharge in under $12 \mathrm{~h}$, but if it takes longer, there are provisions in the procedures for leaving the charger active until recharge is complete.

\section{BEV Testing}

In this section, the procedures specific to $\mathrm{BEV}$ testing are discussed and illustrated with the Nissan Leaf BEV.

\subsection{Legacy SAE J1634 Testing}

At the time of this writing, the SAE-recommended practice for testing BEVs (SAE J1634) is being revised. The original test procedures were outlined in SAE J1634-1995 [9] (originally published in 1995 and revised in 1999). The procedure was simple in its design: for a given drive cycle (only the UDDS and HWY tests were mentioned), drive a succession of cycles from fully charged until the battery is depleted. The range is defined as the point where the vehicle cannot continue driving the required speed trace on the dynamometer. Electric energy consumption was defined by the total AC recharge energy divided by the observed range distance (BEV energy consumption is defined as the energy taken from the wall outlet).

The now-cancelled J1634 procedure has become outdated simply because the modern BEV range is now much higher, owing to dramatic advances in battery technology over the past 20 years. Whereas BEVs from the 1980s and 1990s typically featured a 40- to 50-mile range with lead-acid batteries, today's BEVs will travel over 100 miles on the UDDS cycle, powered by the latest lithium-based batteries.

In terms of test time, determining the UDDS cycle is perhaps the most problematic task. Each cycle is roughly $7.5 \mathrm{mi}$ in length and each cycle consumes 22 minutes. In addition, a 10-min soak period must be included between cycles. The result is a test procedure that could occupy the vehicle, driver, and operator for well over an 8-hour period. And if we consider longer-range BEVs like the Tesla Roadster, the total test time can reach 12-16 hours, which is clearly impractical.

\subsection{Development of New J1634}

In 2008, a new committee was formed to revise J1634. The objectives were to define a shorter, more manageable procedure that provides similar range and electric consumption data compared to the old procedures. In the J1634 revision, a wider variety of test cycles and procedures are addressed, as in the certification tests for conventional vehicles, which have also evolved to include more tests. SAE J-documents are important to the 
automotive industry because certification procedures are usually based upon these consensus documents, which represent the state of the art in test techniques.

The new procedure design had four objectives:

At a minimum, determine range and electricenergy consumption for the UDDS and HWY cycles;

- Do so with a shorter test procedure (in both amount of total test time and number of days to complete);

- Make the procedure elegant and simple; and

- Provide similar results compared to the old procedure.

It became clear that the only way to determine range in a shortened test procedure was not by directly observing it during the test, but by extrapolating the range from test data. This method requires both energy consumption data and the usable battery capacity. The initial idea was to run a separate on-dynamometer battery capacity test, but it was later realized the capacity test and the consumption measurements could be incorporated into a single test procedure.

A simple and repeatable steady-state discharge capacity test is added to cycle tests to help provide the required usable battery energy for range extrapolation. This variable-length cycle is accomplished by running the vehicle at a steadystate speed. After some experimentation, the committee agreed that a speed of $55 \mathrm{mi} / \mathrm{h}$ was a satisfactory compromise between rapid discharge (shortening the test) and realistic discharge rates (if discharge was too fast, thermal management loads could become a significant portion of the battery energy discharge).

Running to the end of capacity under a steadystate speed test has the added benefit of solving a significant problem with the old range determination method-repeatably pinpointing the "end of range." In the old range test, some BEVs (like early lead-acid based BEVs) would gradually lose power and the test would only end during short and infrequent high power demands in the drive cycle. For example, the highest peak power requirement in the UDDS cycle only occurs once per drive cycle, introducing a repeatability problem on the order of 7.5 miles (the length of one UDDS cycle). Running until the vehicle cannot maintain speed in a steady- state cycle offers a much more robust end-of-range point.

\subsubsection{Defining the BEV Test Sequence}

At first, the simplest approach for a combination UDDS, HWY, and battery capacity test was to run a number of UDDS cycles followed by a number of HWY cycles and end with a steady-state drive until end of range. The consumption data for the UDDS and HWY are collected and the total battery capacity recorded (the actual driven range for the test is not useful; the data taken allow for range extrapolations).

Experiments conducted by the committee suggested that it may be important to measure cycle efficiency at both high and low battery state of charge (SOC). The test sequence includes an additional steady-state period in the middle of range in order to collect efficiency data near the end of range. The test procedure sequences still being developed are shown in Figure 1. Both options consist of four UDDS cycles and two HWY cycles.

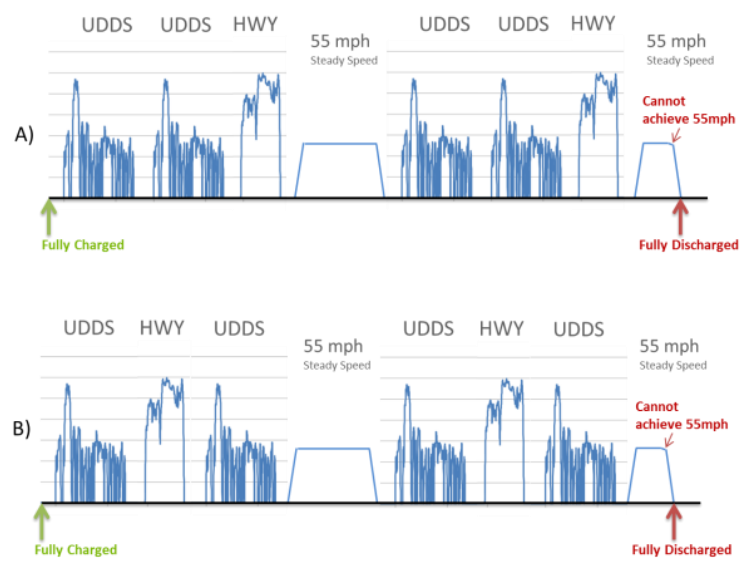

Figure 1: Two BEV testing sequences under development

\subsubsection{Range Extrapolation Method}

The basic equation to extrapolate range is shown below as Equation 1.

$R=\frac{U B E}{E C} \quad$,

where

$R \quad=$ Range (unit distance);

$U B E=$ Usable Battery Energy (DC Wh); and 
$E C=$ Energy Consumption Rate $(\mathrm{DC} \mathrm{Wh} / \mathrm{unit}$ distance).

Extrapolating the range expected from conducting full tests depends upon several issues, one of which is similarly characterizing the electrical-energy consumption. Transient changes in results occur in the first cycle or cycles for any vehicle test, but BEVs have the added effect of reduced regenerative (regen) braking at high SOC levels, reducing the overall energy efficiency of the first cycle. Figure 2 shows the $\mathrm{DC} \mathrm{Wh} / \mathrm{mi}$ of the Nissan Leaf over a full UDDS range test. The first cycle is noticeably higher in consumption than the others. This is due to a combination of additional losses during warm-up but mostly to less regen energy recovery during braking.

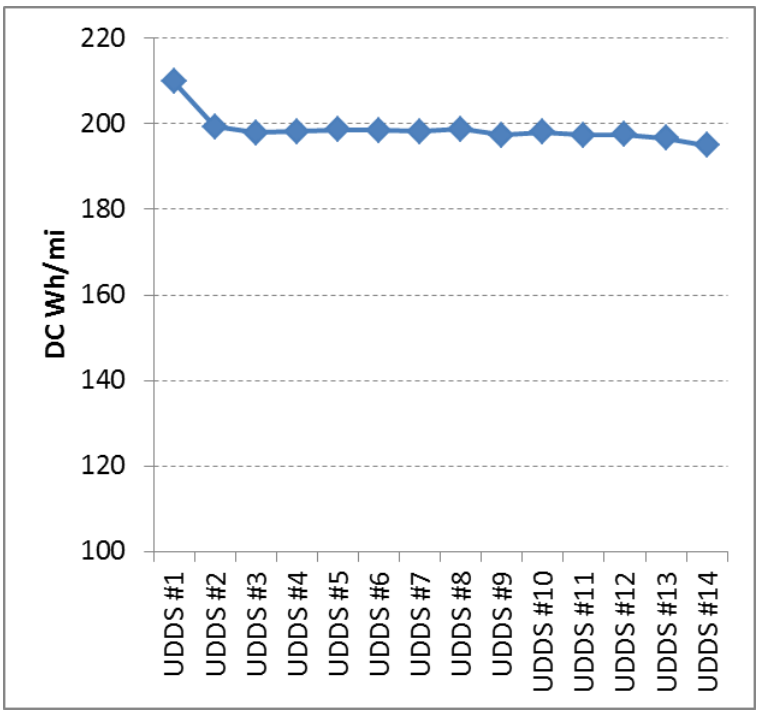

Figure 2: Nissan Leaf energy consumption (DC $\mathrm{Wh} / \mathrm{mi}$ ) for a full range test

Looking back at Figure 1, if the $\mathrm{Wh} / \mathrm{mi}$ is calculated by averaging all four UDDS results, then the first-cycle effect is $25 \%$ of the total, whereas during an actual full range test the effect is only $1 / 14^{\text {th }}(7 \%)$. A solution to this problem is to employ a weighting approach for the first cycle that is equal to the proper fraction of energy represented in the first cycle. This weighting factor for the first cycle is expressed in Equation 2:

$$
W_{U D D S \# 1}=\frac{E C_{U D D S \# 1}}{U B E}
$$

\subsection{Nissan Leaf Test Results}

The Nissan Leaf was tested using the combination UDDS/HWY/Full Depletion test shown in Figure 1A. These results are shown in Table 1. (SS@55= steady-state speed at $55 \mathrm{mph}$ ).

Table1: Nissan Leaf UDDS/HWY/Full Depletion Combination Test Results

\begin{tabular}{|c|c|c|c|c|c|}
\hline Test \# & Cycle & Wh & $\begin{array}{c}\text { Cycle } \\
\text { Dist [mi] }\end{array}$ & $\begin{array}{c}\text { DC } \\
{[\mathrm{Wh} / \mathrm{mi}]}\end{array}$ & $\begin{array}{c}\text { AC } \\
{[\mathrm{Wh} / \mathrm{mi}]}\end{array}$ \\
\hline 71109006 & UDDS & 1583.6 & 7.47 & 211.9 & 251.4 \\
71109007 & UDDS & 1494.0 & 7.47 & 199.9 & 237.1 \\
\hline 71109007 & HWY & 2422.8 & 10.26 & 236.1 & 280.0 \\
71109008 & SS @ 55 & 7844.5 & 30.94 & 253.5 & \\
71109009 & HWY & 2409.4 & 10.25 & 235.2 & 278.9 \\
71109010 & UDDS & 1494.2 & 7.48 & 199.7 & 236.8 \\
71109011 & UDDS & 1468.5 & 7.47 & 196.7 & 233.3 \\
\hline 71109012 & SS @ 55 & 2429.2 & 9.39 & 258.6 & \\
\cline { 4 - 5 } & \multicolumn{7}{|c}{ Total } & 21146.2 & 90.7 &
\end{tabular}

AC Recharge energy into charger $=25.08 \mathrm{AC} \mathrm{kWh}$ $D C$ Recharge energy into battery $=21.64 D C \mathrm{kWh}$

\subsubsection{Leaf Range and Consumption Calculations}

Averaging the highway consumption rate results $(235.6 \mathrm{Wh} / \mathrm{mi})$ and extrapolating out to 21146.2 $\mathrm{Wh}$, the HWY range is found to be 89.7 miles. This is close to the average full range measurement of 90.5 miles.

The UDDS range was calculated using weighting on the first cycle. The first-cycle weighting is equal to $1583.6 \mathrm{Wh}$ divided by the total 21146 Wh, with the remaining UDDS cycles weighted equally. The resulting weighted UDDS consumption rate is $199.8 \mathrm{Wh} / \mathrm{mi}$. The calculated UDDS range with weighting is 105.9 miles. For reference, the non-weighted UDDS consumption is $202.1 \mathrm{Wh} / \mathrm{mi}$ and the range is 104.7 miles. The full UDDS range test result was 106 miles. The weighted approach to range extrapolation indeed predicts a range closer to the directly measured range test.

\subsubsection{Leaf Recharge System Efficiencies}

A number of transportation system analysts were eager to find out our results on charger efficiency for the Leaf as a high-volume BEV benchmark. Studies require this efficiency as an input to estimate grid demands and upstream energy usage [10] from electric energy consumption estimated from vehicle systems models [11]. It became clear in responding to these requests that the 
terminology in the area of recharge system efficiencies is inadequate. "Charger Efficiency" is a term often used, but rarely defined properly in the context of actual test data.

Figure 3 offers some representative Leaf recharge data and some suggestions for useful terms to define the various energy efficiencies in terms of specific measurement points. (EVSE = electric vehicle supply equipment.)

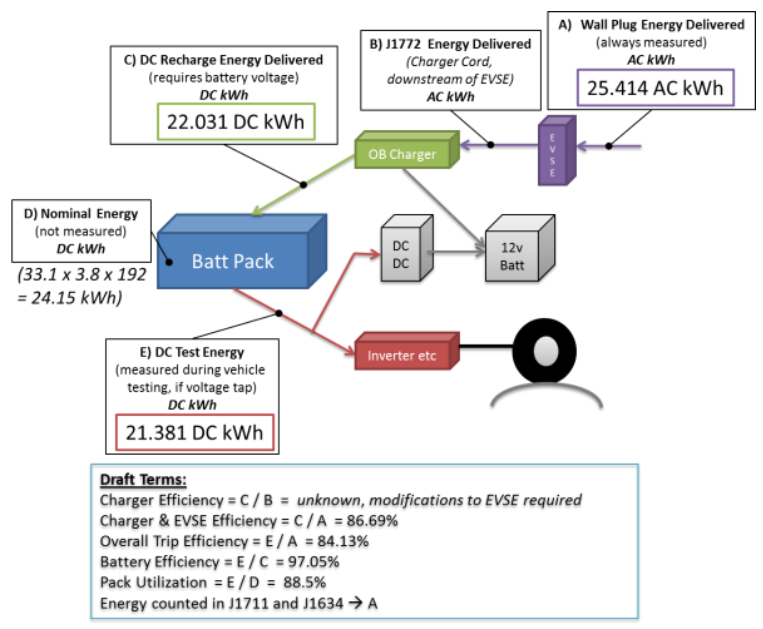

Figure 3: Leaf Recharge System Efficiency Metrics

The most important clarification to be made is differentiating the DC Test Energy from the DC Recharge Energy Delivered. "Charger Efficiency" relates point $\mathrm{B}$ in Figure 3 to point $\mathrm{C}$, whereas to calculate $\mathrm{AC} \mathrm{Wh} / \mathrm{mi}$ from $\mathrm{DC}$ consumption rates (a calculation analysts make), one would need to use the relationship between point $\mathrm{E}$ and point $\mathrm{A}$. Point $\mathrm{A}$ must always be measured for any plug-in vehicle and is the basis for SAE J1634 and J1711 electrical-energy consumption. Consumption includes the nominal energy lost in the EVSE.

\section{PHEV Testing}

With two energy sources used to propel a PHEV, testing is a formidable challenge. If the task were to start a test protocol from scratch, a very direct and appropriate methodology could be designed to accomplish the goals of finding the fuel and electric consumption. However, we have the complicating constraint that legacy cycles and test conditions must be followed as much as possible. The recommended test approaches will be illustrated by looking at the results for two PHEV test vehicles, the Chevy Volt and a PHEV-converted Toyota Prius.

\subsection{Introduction to PHEV Operation}

Before the results are discussed, let us review PHEV operation and fundamental variations in design that affect the testing approach.

One design goal all PHEVs share is the maximization of off-board electrical-energy usage. We must assume that the customer will recharge as often as practical, at a minimum every night after normal usage. How the propulsion system is sized to deplete that energy can vary. If the vehicle has all-electric capability to drive on a particular drive cycle, then the expected results and reporting of the results may be different compared to a PHEV without all-electric drive cycle power capability. The two example vehicles in this paper, the PHEV-converted Prius and the Chevy Volt, represent these two design approaches.

Figure 4 illustrates the operational behavior of two types of PHEV designs. In Figure 4A, the vehicle is fully capable of driving all-electric on the drive cycle; thus the engine is engaged only when the SOC is depleted to a point where the chargesustaining mode is invoked to maintain driving (this has been referred to as an "Extended-Range Electric Vehicle" or "EREV"). Figure 5B shows the engine being used only during short periods of time when the electric drive system is not capable of the power required to drive the cycle (This PHEV type is referred to as "Blended PHEV" because the depleting mode blends engine and electric drive to meet the driving demands).

A)

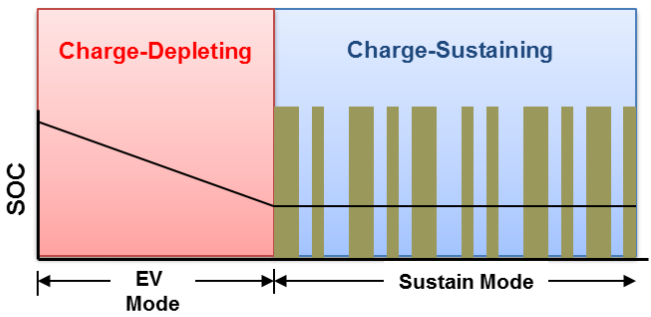

$=$ Engine-on

B)

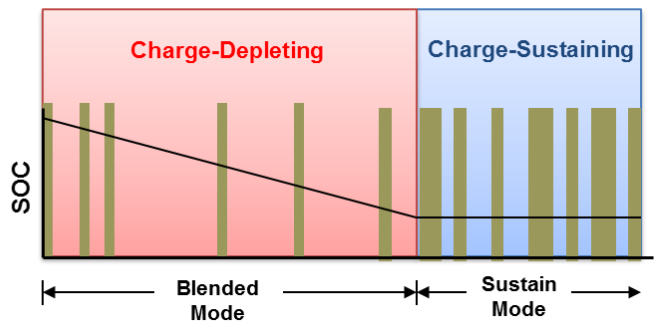

Figure 4: A) Blended and B) EREV PHEV Types 
Both vehicle types are PHEVs, and although the results may be processed or reported differently, the testing protocol for both is essentially identical (this will be explained further in the next section).

\subsection{PHEV Test Method Development}

In 2006, at the same time Argonne started experimenting with PHEV hardware, SAE formed a task force committee to revise SAE J1711. The original J1711 covered both HEVs and PHEVs, but the PHEV section was not well developed because at the time of its writing, there was very little PHEV hardware with which to validate the procedures. The original procedures were developed by conducting thought experiments based upon guesses of how future PHEVs would operate. The new procedures address both blended and EREV types of PHEVs and do so on the basis of test procedure development with real test hardware.

For any given test schedule, the procedures are comprised of two separate test procedures. One is the Full Charge Test (FCT), which captures all charge-depleting-mode fuel and electricity consumption results. The other is the ChargeSustaining Test (CST), which is conducted the same way hybrids have been tested for over a decade. PHEV test procedures also define the steps and requirements for soak and charging for the FCT.

\subsubsection{End-of-Test Criteria}

It may not appear difficult at first, but one of the most challenging issues was a robust determination of the FCT end-of-test (EOT). Two EOT criteria were established in J1711 for the FCT that defined what conditions are to be met to signal that charge-depleting operation has ended. One criterion is based upon the acceptance criteria for charge-sustaining results and the second is based upon the notion that no additional testing is required if the amount of discharge energy from the last cycle is insignificantly small. This level of insignificance is defined as $2 \%$ of the total energy depleted in the FCT (see Figure 5).
1)

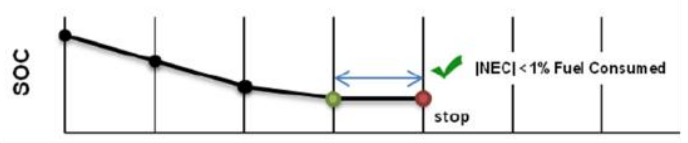

2)

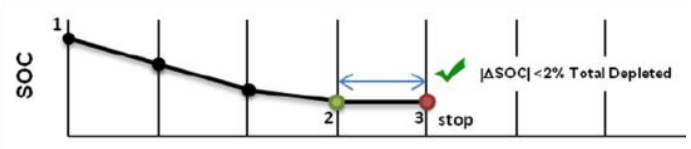

Figure 5: Two End-of-Test Criteria in J1711 $(\mathrm{NEC}=$ net energy change)

The first criterion mirrors long-established chargedepleting test procedures [1]. It states that testing will continue if the net energy change (NEC) is larger than $1 \%$ of the fuel consumed in the cycle. However, this criterion can be particularly difficult to meet during actual PHEV testing, especially for vehicles with large battery packs and potentially large swings in SOC. The rationale for developing the second criterion is based upon the fact that the purpose of the FCT is to capture all the depleting results within an acceptable tolerance (on the order of other practical accuracy expectations). Given that the goal of the FCT is not to find an accurate charge-sustaining result, we should not be bound by a tight charge-sustaining validation criterion. The results of the charge-sustaining tests in the FCT are not part of the final results. We decide to end testing not when we observe a chargesustaining test, but when we observe the practical end of charge-depleting behavior. This is an important distinction that will become evident when PHEV results are analyzed later in this paper.

\subsubsection{PHEV Range Definitions}

A few PHEV range definitions were developed in order to satisfy a number of stakeholders interested in describing PHEVs or differentiating them from one another. The definitions are useful for manufacturers and regulators. A robust analytical technique was needed to pinpoint the end of charge-depleting behavior. However, these points are not necessarily evident from looking at the instantaneous data. Techniques were established, some based upon the summary data from each test in the FCT.

The "All-Electric Range" (AER) is defined as the distance driven during the FCT before the engine is first engaged. The AER is different from the transition point from depleting to sustaining, which is not very clear or distinct in the data. After engine start, the vehicle may continue to discharge, 
or it could charge back up to a level higher than that of the engine start level.

The "Equivalent All-Electric Range" (EAER) is useful to describe the full capability of the PHEV in terms of AER. The EAER can help provide a way to compare EREV and blended-type PHEVs using a range metric to describe relative battery capacities.

The EOT criterion tells the test operators when to stop testing but also provides a clear and robust point to separate depleting- from sustainingmode cycles. The general trend in an FCT is depleting cycles, followed by the transition cycle, followed by the cycles that are not significantly charge-depleting. The charge-depleting cycle range $\left(R_{c d c}\right)$ is the distance traveled in all the charge-depleting cycles, including the transition cycle.

The actual point within the transition cycle where the mode changes is not easily found by direct analysis of the transient data but is inferred in methods such as the Actual Charge-Depleting Range $\left(\mathrm{R}_{\mathrm{cda}}\right)$ calculation. The equations for specific range definitions are detailed in the results section later in this paper to better illustrate the methods.

\subsubsection{Hot/Cold Weighting Incompatibility}

Every effort was made to design the FCT to adhere to legacy procedures and conditions. One important incompatibility is the hot and cold weighting described in Section 2.1. It was concluded that there is no rational method for applying hot/cold weighting to the UDDS FCT. For EREV PHEVs, it is not entirely rational to weight the first cycle differently from the others on the basis of the original reasoning behind weighting "cold-start" effects. How would the rest of the cycles in the FCT be treated? It became clear that any attempt to match the original hot/cold weighting procedures would be somewhat arbitrary and would not provide additional meaning to the results. Criteria emissions procedures do, however, include a weighting approach directed to engine start to address legacy emissions regulations [12].

\subsection{Chevy Volt Results}

In this section, the PHEV test procedures and post-processing will be illustrated with the Chevy Volt on the UDDS FCT.

\subsubsection{Volt FCT Data}

The Volt was recharged to full while soaking overnight before being given a UDDS FCT the next day. Because this was research testing, cycles were repeated until the stricter of the two EOT tolerances was met. Table 2 shows the Chevy Volt UDDS FCT results. Cycle 9 does meet the second criterion, but 10 cycles were required to achieve the most stringent " $1 \%$ of the fuel usage" criterion. If the second EOT criterion were used, only nine cycles would be required to complete the UDDS FCT.

Table 2: Chevy Volt UDDS FCT Results

\begin{tabular}{|c|c|c|c|c|c|c|c|}
\hline \multirow[b]{2}{*}{ Cycle } & \multirow[b]{2}{*}{ Miles } & \multirow[b]{2}{*}{$\begin{array}{l}\text { MPG } \\
\text { actual }\end{array}$} & \multirow[b]{2}{*}{$\begin{array}{c}\text { Ah x } \\
(\mathrm{Vi}+\mathrm{V} f) \\
/ 2\end{array}$} & \multicolumn{2}{|c|}{ EOT Criteria } & \multicolumn{2}{|c|}{ AC Wh Calcs } \\
\hline & & & & $\begin{array}{l}\text { (1) } \Delta \% \\
\text { of Fuel }\end{array}$ & $\begin{array}{l}\text { (2) } \Delta \% \\
\text { of Disch }\end{array}$ & $\begin{array}{l}\text { Total \% } \\
\text { of Disch }\end{array}$ & $\begin{array}{c}\mathrm{AC} \\
\mathrm{Wh} / \mathrm{mi}^{1}\end{array}$ \\
\hline 1 & 7.43 & $\inf$ & 1582.9 & $25.72 \%$ & -- & $14.47 \%$ & 255.3 \\
\hline 2 & 14.86 & $\inf$ & 1535.7 & $25.22 \%$ & $49.24 \%$ & $14.04 \%$ & 247.4 \\
\hline 3 & 22.29 & inf & 1521.0 & $25.33 \%$ & $32.78 \%$ & $13.91 \%$ & 245.1 \\
\hline 4 & 29.73 & $\inf$ & 1515.2 & $25.61 \%$ & $24.62 \%$ & $13.85 \%$ & 244.2 \\
\hline 5 & 37.16 & inf & 1505.6 & $25.75 \%$ & $19.65 \%$ & $13.76 \%$ & 242.7 \\
\hline 6 & 44.59 & $\inf$ & 1506.1 & $26.12 \%$ & $16.43 \%$ & $13.77 \%$ & 242.6 \\
\hline 7 & 52.03 & 232.4 & 1267.6 & $22.44 \%$ & $12.15 \%$ & $11.59 \%$ & 204.2 \\
\hline 8 & 59.47 & 60.6 & 386.5 & $6.95 \%$ & $3.57 \%$ & $3.53 \%$ & 62.2 \\
\hline 9 & 66.90 & 51.0 & 86.2 & $1.56 \%$ & $0.79 \%$ & $0.79 \%$ & 13.9 \\
\hline 10 & 74.33 & 49.0 & 31.3 & $0.57 \%$ & $0.29 \%$ & $0.29 \%$ & 5.0 \\
\hline
\end{tabular}

${ }^{1}$ Based upon 13.102 AC kWh recharge to full

The NEC is in the column labelled " $A h x(V i+$ $V f) / 2$ " (it is also referred to in $\mathrm{J} 1711$ as "SOC"). Voltage is taken under no current load at the beginning and end of each cycle test ( $V i$ and $V f$, respectively). It is used in the EOT criterion and in determining the transition cycle, $\mathbf{R}_{\text {cdc }}$, and $\mathbf{R}_{\text {cda }}$.

Other data shown in Table 2 are the calculations of the $\mathrm{AC} \mathrm{Wh} / \mathrm{mi}$ on the basis of the NEC DC results. Each cycle is given a percentage contribution to the total discharge (based upon the NEC) and then the total recharge energy is parsed among these cycles on the basis of their percentage contribution. A recharge energy of 13.102 AC $\mathrm{kWh}$ was divided among the FCT cycles, resulting in charge-depleting energy consumption of 242 to $255 \mathrm{AC} \mathrm{Wh} / \mathrm{mi}$ (seen in the last column of Table 2). 


\subsubsection{Volt All-Electric Range and Equivalent All-Electric Range}

Using the simple definition of AER from Section 4.2.2, the Volt UDDS AER was found to be 50.55 miles. The first engine start on the FCT occurred 5.96 miles into the $6^{\text {th }}$ cycle (Table 2).

The Volt continues to deplete a noticeable amount of energy after the first engine start before charge-sustaining behavior is observed. In this case, the EAER would then be expected to be longer than the AER. The EAER is calculated using Equation 3 below:

$E A E R=R_{c d c} \times\left(F C_{c s}-F C_{c d}\right) / F C_{c s}$,

where

$R_{c d c}=$ Charge-depleting cycle range, from beginning of test to the end of the last depleting cycle according to the EOT tolerance criterion;

$\mathrm{FC}_{\mathrm{cs}}=$ Fuel consumption rate (fuel/distance) in charge-sustaining mode (chargebalanced); and

$\mathrm{FC}_{\mathrm{cd}}=$ Fuel consumption rate (fuel/distance) for the charge-depleting cycles (chargebalanced).

For the Volt, the EAER for the UDDS cycle is $51.86 \mathrm{mi}$.

\subsubsection{Volt Charge-Depleting Range}

The Volt's actual charge-depleting range $\left(\mathrm{R}_{\text {cda }}\right)$ is calculated using the definition in J1711. The second EOT criterion in J1711 is also helpful in solving for $\mathrm{R}_{\text {cda }}$. Ending the FCT at cycle 8 means that cycle 7 must be the transition cycle. The J1711 charge-depleting range definition is insensitive to instantaneous rises and drops in SOC within any given cycle; the calculations were written to consider only the test cycle endpoints. The method is detailed in Equations 4 and 5 and expressed graphically in Figure 6.

$$
Z_{n}=\frac{\Delta S O C_{n}}{\Delta S O C_{n-1}}
$$

and

$$
R_{c d a}=R_{n-1}+\left(Z_{n} \times D_{n}\right) \quad,
$$

where

$\triangle S O C=$ Defined as $\triangle A h x(V i+V f) / 2$;

$\mathrm{Z}_{\mathrm{n}} \quad=$ Depleting fraction of transition cycle; $n=$ Transition cycle (cycle 7);

$D_{n} \quad=$ Measured cycle distance of cycle $\mathrm{n}$; and

$R_{n-1}=$ Measured distance from the start of FCT to the end of cycle $n-1$.

Substituting test results into equations 4 and 5, the calculations to find the UDDS FCT $R_{c d a}$ are as follows:

$Z_{n}=386.5 / 1267.6=0.3049 ;$

$R_{c d a}=52.03+(0.3049 \times 7.440)=54.30 \mathrm{mi}$.

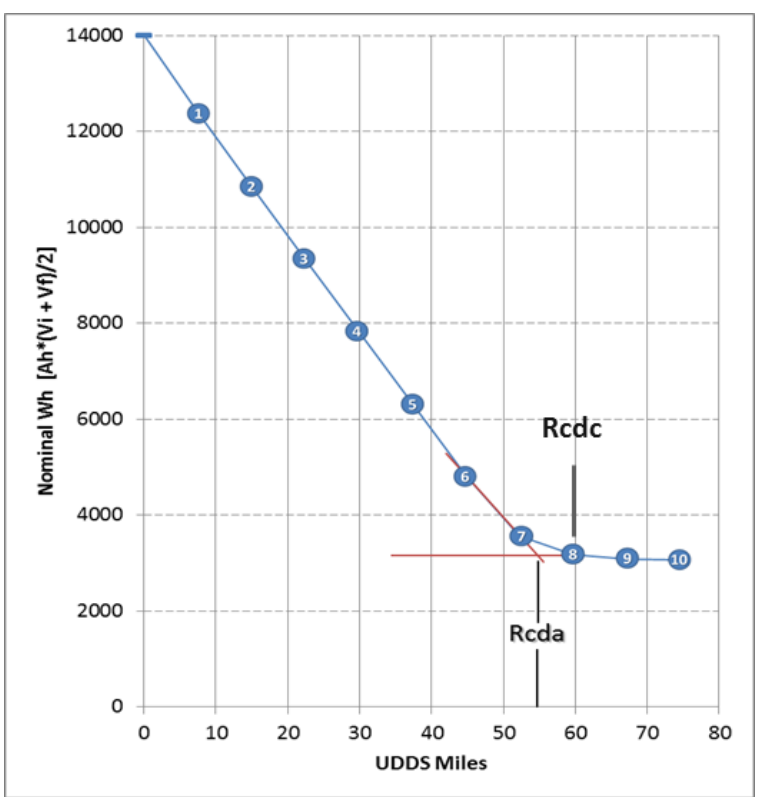

Figure 6: Determining the UDDS Charge-Depleting Ranges for the Chevy Volt

\subsubsection{Volt Charge-Depleting Results}

We can describe the charge-depleting results by lumping together all the results within the chargedepleting cycle range. This includes both electricity and fuel consumption rates, as shown in Equations 6 and 7:

For electricity consumption,

$E C_{C D}=E_{\text {TotalAC }} / R_{c d c}$

and

$\begin{aligned} E C_{C D} & =13102 \mathrm{AC} \mathrm{Wh} / 59.47 \mathrm{mi} \\ & =220.3 \mathrm{AC} \mathrm{Wh} / \mathrm{mi}\end{aligned}$

For fuel consumption,

$F C_{C D}=$ Fuel $_{\text {Total }} / R_{c d c}$ 
and

$$
\begin{aligned}
F C_{C D} & =0.0026105 \mathrm{gal} / 59.47 \mathrm{mi} \\
& =0.004389 \mathrm{gal} / 100 \mathrm{mi} .
\end{aligned}
$$

While the above results are technically accurate, there may be a better approach that is more in line with the expectations of an EREV. The charge-depleting mode of the Volt is essentially electric-only. Another way to describe depleting operation is to take all the electrical energy consumed and divide it by an appropriate range, in this case the EAER. These results are shown in Equation 8:

$$
E C_{C D}=E_{\text {TotalAC }} / \text { EAER . }
$$

Using the test results, the calculations are

$$
\begin{aligned}
E C_{C D} & =13102 \mathrm{AC} \mathrm{Wh} / 51.86 \mathrm{mi} \\
& =252.6 \mathrm{AC} \mathrm{Wh} / \mathrm{mi} .
\end{aligned}
$$

\subsubsection{Volt Charge-Sustaining Results}

Whereas charge-sustaining hybrids with small battery packs typically do not have trouble meeting the charge-sustaining criteria, PHEVs present new challenges for testing. The results in Table 3 show Volt charge-sustaining UDDS results that were within the charge-sustaining tolerance. Multiple UDDS prep cycles the day before testing were required to achieve steady charge balance.

Table 3: Volt UDDS CST Results

\begin{tabular}{|c|c|c|c|c|}
\hline Cycle & Miles & $\begin{array}{c}\text { MPG } \\
\text { actual }\end{array}$ & $\begin{array}{c}\text { Ah } \mathbf{x} \\
\mathbf{( V i + V f )} \\
\mathbf{/ 2}\end{array}$ & $\begin{array}{c}\Delta \% \\
\text { of Fuel }\end{array}$ \\
\hline 1 & 7.44 & 44.9 & 47.59 & $0.85 \%$ \\
\hline 2 & 7.43 & 47.0 & -27.47 & $-0.51 \%$ \\
\hline Comb & & & 20.12 & $0.18 \%$ \\
\hline $43 / 57$ & & 46.1 & & \\
\hline
\end{tabular}

\subsection{PHEV-Converted Prius Results}

Many of the PHEV test procedures were validated using charge-sustaining hybrids converted to PHEVs. These vehicles operate in a "blended" depleting mode where both the engine and the battery are consuming energy. This operation was not originally considered during the development of J1711 in the 1990s. The challenge in developing the J1711 update was to design one procedure that tested both EREVs and blended PHEVs without bias or inconsistencies.

The test vehicle in this section was a secondgeneration Prius with a PHEV kit installed by an aftermarket manufacturer.

\subsubsection{PHEV-Converted Prius FCT Data}

The UDDS FCT was run in the same manner as for the Volt; however the EOT criteria were not fully developed at the time of testing (testing occurred in 2007). Depleting mode was generally observed by the operators in cycle 6 and the test was terminated. Recent analysis shows that although

\begin{tabular}{|c|c|c|c|c|c|c|c|c|}
\hline & \multirow[b]{2}{*}{ Miles } & \multirow[b]{2}{*}{$\begin{array}{c}\text { MPG } \\
\text { actual }\end{array}$} & \multirow[b]{2}{*}{$\begin{array}{c}\text { DC } \\
\text { Wh/ } \\
\mathrm{mi}\end{array}$} & \multirow[b]{2}{*}{$\begin{array}{c}\text { Ah } x \\
(V i+V f) \\
/ 2\end{array}$} & \multicolumn{2}{|c|}{ EOT Criteria } & \multicolumn{2}{|c|}{ AC Wh Calcs } \\
\hline & & & & & $\begin{array}{l}\text { (1) } \Delta \% \\
\text { of Fuel }\end{array}$ & \begin{tabular}{|c|} 
(2) $\Delta \%$ \\
of \\
Disch
\end{tabular} & $\begin{array}{c}\text { Total \% } \\
\text { of } \\
\text { Disch }\end{array}$ & $\begin{array}{c}\text { AC } \\
\text { Wh/mi }\end{array}$ \\
\hline 1 & 7.46 & 154.1 & 139.1 & 1104.7 & $26.3 \%$ & -- & $23.7 \%$ & 174.0 \\
\hline 2 & 7.47 & 207.3 & 142.3 & 1120.5 & $26.7 \%$ & $50.4 \%$ & $24.0 \%$ & 176.3 \\
\hline 3 & 7.47 & 210.8 & 143.0 & 1124.4 & $26.8 \%$ & $33.6 \%$ & $24.1 \%$ & 177.0 \\
\hline 4 & 7.46 & 218.2 & 140.0 & 1102.8 & $26.3 \%$ & $24.8 \%$ & $23.6 \%$ & 173.7 \\
\hline 5 & 7.47 & 64.7 & 8.8 & 129.2 & $3.08 \%$ & $2.82 \%$ & $2.8 \%$ & 20.3 \\
\hline 6 & 7.47 & 64.0 & 3.0 & 82.7 & $1.97 \%$ & $1.77 \%$ & $1.8 \%$ & 13.0 \\
\hline
\end{tabular}
the first EOT criterion was not met, the second EOT criterion was satisfied in cycle 6 . The FCT data are shown in Table 4.

Table 4: PHEV-Converted Prius UDDS FCT Results

The data listed in Table 4 and plotted in Figure 7 show that the second EOT criterion can be robust in locating the transition point and provides accurate guidance for EOT without making the test burdensome.

\subsubsection{PHEV-Converted Prius Charge- Depleting Range}

Again using the second EOT criterion as a guide to finding the transition cycle, the UDDS $\mathrm{R}_{\mathrm{cda}}$ can be calculated using Equations 4 and 5. The results are shown graphically in Figure 7.

$$
\begin{aligned}
& Z_{n}=129.2 / 1102.8=0.1172 \\
& R_{c d a}=29.86+(0.1172 \times 7.47)=30.74 \mathrm{mi}
\end{aligned}
$$




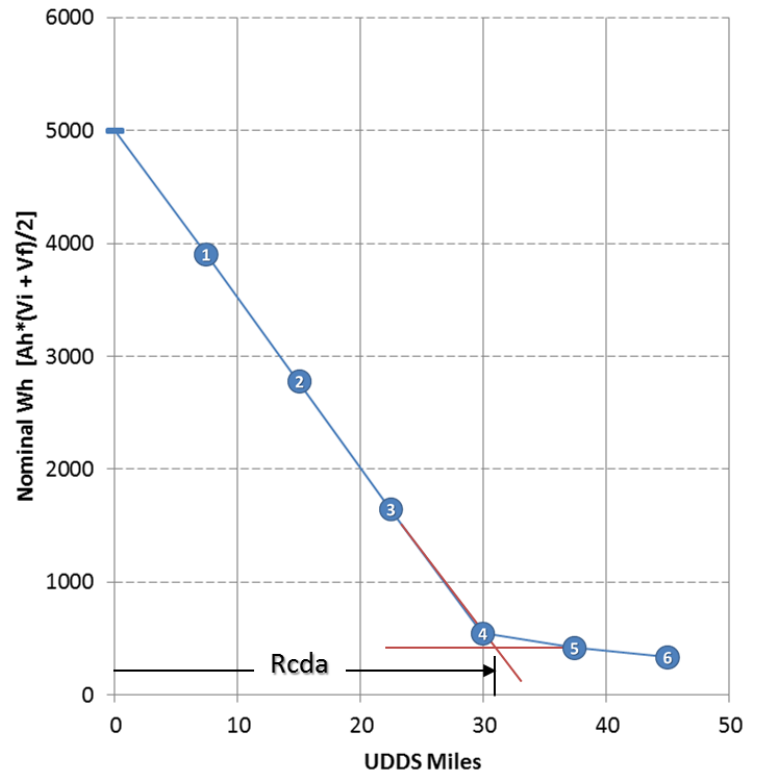

Figure 7: Determining the UDDS Charge-Depleting Range of the PHEV-Converted Prius

\subsubsection{PHEV-Converted Prius Charge- Depleting Results}

Describing blended depleting operation requires that both the fuel and electric energy consumption rates be calculated and reported. Lumping all the depleting cycle results together gives the fuel and electric consumption shown below.

$$
\begin{aligned}
E C_{C D} & =5481 \mathrm{AC} \mathrm{Wh} / 7.33 \mathrm{mi} \\
& =146.82 \mathrm{AC} \mathrm{Wh} / \mathrm{mi} \\
F C_{C D} & =0.15407 \mathrm{gal} / 37.33 \mathrm{mi} \\
& =0.4127 \mathrm{gal} / 100 \mathrm{mi}
\end{aligned}
$$

As this is a blended PHEV, it would be inappropriate to describe the depleting mode in terms of electric-only consumption. What is needed is a method to describe the chargedepleting results in terms of the actual chargedepleting range. This is handled according to the following methodology:

The electrical-energy consumption is straightforward and calculated as

$$
\begin{aligned}
E C_{C D} & =5481 \mathrm{AC} \mathrm{Wh} / 30.74 \mathrm{mi} \\
& =178.3 \mathrm{AC} \mathrm{Wh} / \mathrm{mi} .
\end{aligned}
$$

Calculating the fuel usage associated only with the charge-depleting range requires extrapolation of trends similar to that used to calculate $\mathrm{R}_{\text {cda. }}$. To calculate all the fuel consumed within $\mathrm{R}_{\mathrm{cda}}$, all the fuel consumed in the cycles before the transition cycle is combined. Then the fuel in the transition cycle is calculated by multiplying the fraction $Z_{n}$ (from Equation 4) by the fuel consumed in the cycle before the transition cycle (cycle $n-1$ ). The rationale is that the portion of the transition cycle in depleting mode is more similar to the cycle $n-1$ than it is to the charge-sustaining cycle. The calculation is given in Equation 9:

$$
\begin{aligned}
& F C_{C D}=\frac{\left[\left(\sum_{i=1}^{n-1} Y_{i}\right)+Z_{n} \times Y_{n-1}\right]}{R_{c d a}} \\
& \begin{aligned}
F C_{C D} & =[0.154 \mathrm{gal}+0.1172 \times 0.1542 \mathrm{gal}] / 30.74 \\
& =0.0055998 \mathrm{gal} / \mathrm{mi}
\end{aligned} \\
& \text { or } \quad
\end{aligned}
$$

178.6 MPG

\subsubsection{PHEV-Converted Prius Charge- Sustaining Results}

The UDDS charge-sustaining results for the PHEV-converted Prius ran into SOC balance issues. The add-on kit can sometimes interfere with the ability of the vehicle to adapt its control strategy to maintain charge balance over a cycle. The measured fuel economy results in Table 5 show a slight positive energy removed from the battery pack and thus reflect a higher MPG than SOC-balanced cycles would yield. The sustaining test results were corrected using trends from other tests that reveal the relationship between NEC and marginal differences in fuel consumption.

Table 5: PHEV-Converted Prius UDDS CST Results

\begin{tabular}{|c|c|c|c|c|c|}
\hline Cycle & Miles & $\begin{array}{c}\text { MPG } \\
\text { actual }\end{array}$ & $\begin{array}{c}\text { Ah x } \\
\mathbf{( V i + V f )} \\
\mathbf{/ 2}\end{array}$ & $\begin{array}{c}\Delta \% \\
\text { of Fuel }\end{array}$ & $\begin{array}{c}\text { SOC- } \\
\text { Corrected } \\
\text { MPG }\end{array}$ \\
\hline 1 & 7.47 & 56.8 & 51.65 & $1.16 \%$ & 55.05 \\
\hline 2 & 7.47 & 63.3 & 60.73 & $1.53 \%$ & 60.77 \\
\hline Comb & & & 112.38 & $1.33 \%$ & \\
\hline $43 / 57$ & & 60.3 & & & 58.17 \\
\hline
\end{tabular}

\section{Utility Factors}

The distance driven in a given day is expected to produce only slight changes in fuel efficiency in conventional vehicles. The hot/cold weighting values in Equation 1 reflect in-use driving habits related to how long drivers leave their vehicle at rest before the next start. The expectations of fuel 
use for a PHEV are highly dependent upon the distance driven in a given day (distance between charge events). Driving data can be a guide to help further analyze the charge-depleting and charge-sustaining results and weight them accordingly.

\subsection{PHEV Utility Factor Analysis}

The Utility Factor (UF) weighting approach was carried over from the original J1711 and updated with new transportation survey data [13]. The UF analysis of test results shows the percentage (based upon driving distance) weighting of the depleting mode compared to the sustaining mode. This weighting is calculated on the basis of the charge-depleting test results matched up to surveys of drivers describing their use of personal vehicles across the United States (of course, the method can be re-evaluated for other countries if a large, representative dataset of daily driving distances is available).

The UF approach requires using the entire DOT National Household Travel Survey (NHTS) database of personal-vehicle usage data to calculate the fraction of depleting-mode driving for any PHEV from 1 to 400 miles of depleting range. This numerically intense approach is required because any analysis applying average usages would be meaningless. For example, if an EREV has a 50-mile electric-only range and it is shown that the average daily driving distance is 37 miles, of course it is incorrect to predict that, if vehicles are recharged every day, all the vehicle-miles in a large fleet will be driven electric-only. However, by analyzing all the daily driving data, one can find that the percentage of daily miles driven all-electric by an entire fleet of 50-mile all-electric PHEVs will be $68.9 \%$.

The most robust UF approach is actually a detailed calculation method that weights each charge-depleting cycle result independently. Each successive cycle has less weighting. Ending with the last charge-depleting cycle, the remaining weighting (one minus the sum of the charge-depleting weights) is assigned to the charge-sustaining results.

For the Volt UDDS FCT, the results and their respective weightings are shown in Table 6.
Table 6: Volt UDDS Results with the Associated Utility Factor Weightings

\begin{tabular}{|c|c|c|c|}
\hline Cycle & $\begin{array}{c}\text { MPG } \\
\text { actual }\end{array}$ & $\begin{array}{c}\text { AC } \\
\text { Wh/mi }\end{array}$ & $\begin{array}{c}\text { UF } \\
\text { Fraction }\end{array}$ \\
\hline 1 & inf & 255.3 & 0.176 \\
2 & inf & 247.4 & 0.141 \\
\hline 3 & inf & 245.1 & 0.113 \\
\hline 4 & inf & 244.2 & 0.091 \\
\hline 5 & inf & 242.7 & 0.073 \\
\hline 6 & inf & 242.6 & 0.059 \\
\hline 7 & 232.4 & 204.2 & 0.048 \\
\hline 8 & 60.6 & 62.2 & 0.040 \\
\hline \multicolumn{7}{|c}{ Charge-sustaining MPG $=$} & 46.1 \\
\end{tabular}

Taking all the results and the UF weighting segments, we obtain a final UF-weighted result of 154.47 MPG and 174.20 AC Wh/mi. This result represents all depleting and sustaining operations in the UDDS cycle.

Similarly, the converted Prius PHEV results with their respective weightings are shown in Table 7 below.

Table 7: PHEV-Converted Prius UDDS Results with the Associated Utility Factor Weightings

\begin{tabular}{|c|c|c|c|}
\hline Cycle & $\begin{array}{c}\text { MPG } \\
\text { actual }\end{array}$ & $\begin{array}{c}\text { AC } \\
\text { Wh/mi }\end{array}$ & $\begin{array}{c}\text { Fractional } \\
\text { UF }\end{array}$ \\
\hline 1 & 154.1 & 174.0 & 0.176 \\
2 & 207.3 & 176.3 & 0.141 \\
3 & 210.8 & 177.0 & 0.113 \\
4 & 218.2 & 173.7 & 0.091 \\
\hline 5 & 64.7 & 20.3 & 0.073 \\
\hline 6 & 64.0 & 13.0 & 0.059 \\
\hline
\end{tabular}

$\begin{aligned} \text { 1-sum(UF) } & =0.347 \\ \text { Charge-sustaining MPG } & =58.2\end{aligned}$

Taking the same approach, the UF-weighted results for the PHEV-converted Prius are 92.63 MPG and $93.46 \mathrm{AC} \mathrm{Wh} / \mathrm{mi}$.

In this paper, only the PHEV results of the UDDS cycle were used for illustrations of the calculation methods. All relevant drive cycles can be tested according to the same approach. EPA fuel economy labeling methods use data from all five tests listed in Section 2 to represent real-world expectations of fuel use. Many researchers are hard at work looking into ways to take UF-weighted 
PHEV cycle results and make real-world predictions of both fuel and electricity usage in PHEVs. As more in-use data and better driving statistics become available, robust calculation methods may follow.

\section{Conclusions}

In order to provide answers about the efficiency of new PEVs, appropriate test procedures must be developed that are robust and compatible with the technology and how people use it. The process took several years with many experts participating in the task forces. BEV and PHEV test procedures were tested with a Nissan Leaf, a PHEV-converted Prius and a Chevy Volt with much success. Major findings of the validation are:

- The new BEV test sequence does save considerable time and can reproduce range and efficiency results of the much longer old methods

- First cycle effects of BEVs can be appropriately weighed in order to better match old methods.

- New required instrumentation works reliably and if access to voltage is too problematic, the bus voltage is adequate to perform the testing (Volt testing).

- Two EOT criteria are presented. The second criterion appears to provide more robust indication of the transition cycle and when to stop testing.

- Depending upon what type of PHEV is tested, there are specific choices in what charge-depleting range definitions are appropriate and how the final results shall be presented.

- Utility factor calculations allow direct comparisons that are not affected by design or operation among PHEV types.

\section{Acknowledgments}

This work was supported by DOE's Vehicle Technology Office under the direction of Lee Slezak. The submitted manuscript has been created by UChicago Argonne, LLC, Operator of Argonne National Laboratory ("Argonne"). Argonne, a U.S. Department of Energy Office of Science laboratory, is operated under Contract No. DE-AC0206CH11357. The U.S. Government retains for itself, and others acting on its behalf, a paid-up nonexclusive, irrevocable worldwide license in said article to reproduce, prepare derivative works, distribute copies to the public, and perform publicly and display publicly, by or on behalf of the Government.

\section{References}

[1] J1711 ref

[2] SAE J1634: Electric Vehicle Energy Consumption and Range Test Procedure, SAE International, April 1, 1999.

[3] U.S. Code of Federal Regulations, 40 CFR Part 600 -- Fuel Economy of Motor Vehicles

[4] Fuel Economy Labeling of Motor Vehicles: Revisions to Improve Calculation of Fuel Economy Estimates, US EPA, EPA420-R-06017, December 2006.

[5] M. Duoba, et al., Investigating Vehicle Fuel Economy Robustness of Conventional and Hybrid Electric Vehicles, International Electric Vehicle Symposium (EVS21), 2005.

[6] Hioki,

http://www.hioki.com/product/3193/index.ht ml, accessed on 2012-02-29

[7] Hioki, http://www.hioki.com/product/3390/index.ht $\underline{\mathrm{ml}}$, accessed on 2012-02-29

[8] Hioki, http://www.hioki.com/product/339010/33901 0o.html, accessed on 2012-02-29

[9] SAE J1634: Electric Vehicle Energy Consumption and Range Test Procedure, SAE International, April 1, 1999.

[10] Argonne National Laboratory, http://greet.es.anl.gov/, accessed on 2012-0229

[11] Argonne National Laboratory, http://www.autonomie.net, accessed on 201202-29

[12] California Exhaust Emissions Standards and Test Procedures for 2009 and Subsequent Model Zero-Emissions Vehicles and Hybrid Electric Vehicles, in th Passenger Car, LightDuty Truck and Medium-Duty Truck Classes, California Environmental Protection Agency Air Resources Board, December 17, 2008

[14] National Household Travel Survey (NHTS), U.S. Department of Transportation, http://nhts.ornl.gov/index.shtml 


\section{Authors}

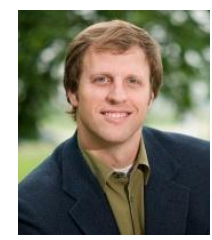

Mr. Michael Duoba

Argonne National Laboratory,

9700 S. Cass, Argonne, IL, 60439, USA

Tel: 630-252-6398 Fax: 630-252-3443

E-mail: mduoba@anl.gov

URL: http://www.transportation.anl.gov/

Lead engineer for Argonne's Advanced

Powertrain Research Facility. He was the chair of SAE J1711 (HEV/PHEV Testing) and a member of SAE J1634 (BEV Testing). He holds an M.S. degree in $\mathrm{ME}$ from the University of Wisconsin-Madison.

Dr. Henning Lohse-Busch

Argonne National Laboratory,

9700 South Cass, Argonne, IL, 60439, USA

Tel: 630-252-9615 Fax: 630-252-

3443

E-mail: hlb@anl.gov

Research Engineer at Argonne's Advanced Powertrain Research Facility.

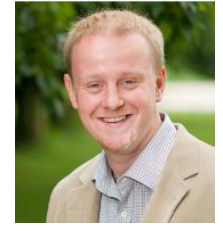

His areas of expertise are advanced technology and alternative fuel vehicle testing and component testing in a vehicle system environment. He holds a $\mathrm{PhD}$ in ME from Virginia Polytechnic Institute and State University.

\section{Mr. Eric Rask}

Argonne National Laboratory,

9700 South Cass, Argonne, IL, 60439, USA

Tel: 630-252-3110 Fax: 630-252-

3443

E-mail: erask@anl.gov

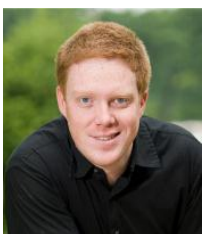

Research Engineer in Argonne's Advanced Powertrain Research Facility. $\mathrm{He}$ is a member of the SAE J1711 (HEV/PHEV Testing) and SAE J1634 (BEV) revision committees. He holds an M.S. degree in ME from University of Michigan-Ann Arbor. 